\title{
Technologies for the integration of miniaturised silicon sensor systems in fibre-reinforced composites
}

\author{
Benjamin Arnold ${ }^{1}$, Ricardo Decker ${ }^{2}$, Florian Rost ${ }^{3)}$, Angelika Bauer ${ }^{2)}$, Jan Mehner ${ }^{1)}$, Lothar Kroll $^{2)}$, \\ Sven Rzepka ${ }^{3)}$, Thomas Otto ${ }^{3)}$
}

1)Department of Microsystems and Biomedical Engineering, benjamin.arnold@etit.tu-chemnitz.de, jan.mehner@etit.tu-chemnitz.de, Chemnitz University of Technology, Reichenhainer Straße 70, 09126 Chemnitz, Germany

2) Department of Lightweight Structures and Polymer Technology, ricardo.decker@mb.tuchemnitz.de, angelika.bauer@mb.tu-chemnitz.de, lothar.kroll@mb.tu-chemnitz.de, Chemnitz University of Technology, Reichenhainer Straße 31/33, 09126 Chemnitz, Germany

3) Fraunhofer Institute for Electronic Nano Systems ENAS, florian.rost@enas.fraunhofer.de, sven.rzepka@enas.fraunhofer.de, thomas.otto@enas.fraunhofer.de, Micro Materials Center, Technologie-Campus 3, 09126 Chemnitz, Germany

\section{Keywords}

CNT, functional integration, MEMS, micro injection moulding, SHM

\begin{abstract}
Functional integration processes gain more and more importance in lightweight engineering. In this paper we discuss how to improve fibre-reinforced composites with structurally integrated condition monitoring systems, suitable for predicting failure behaviour. Especially commercially available and tested silicon sensors, but also new developments are well-suited for this intention. We present a smart semi-finished textile with integrated silicon sensors for in-situ conditions and process monitoring in fibre-reinforced composites. It consists of a textile substrate tape with integrated electrically conductive fibres and various silicon sensors, applied by micro-injection moulding. A so-called "interposer" is used as an electrical adapter between the microstructures of the sensor system and the mesostructures of the textile. The key technology used for the encapsulation and electrical contacting of the sensor nodes is a two-stage two-component micro injection moulding process, allowing for a cost efficient and application specific mass production. As proof of concept we chose the injection moulding process to investigate the influence of the fabrication process on all electronic components with a silicon stress measurement chip. We performed in-situ measurements of temperature and inplane mechanical stress for different glass fibre contents of the PA6 melt and tool temperatures and compared the results with a finite element simulation.
\end{abstract}

\section{Introduction}

\subsection{Motivation}

The importance of high-performance fibre-reinforced composites used in lightweight engineering for complex highly loaded structures is rapidly increasing worldwide [1-2]. To protect them from unpredictable failure behaviour such as fibre cracking or delamination, structures are currently oversized in most cases - not compliant to the idea of lightweight design. The main objective of "MERGE Technologies for Multifunctional Lightweight Structures" is to improve lightweight engineering with energy and resource efficient hybrid structures suitable for large-scale production. Therefore, it is essential to establish structurally integrated condition monitoring systems [1-5]. 


\subsection{Integration concept}

The intention of our investigation was the integration of sensor systems into fibre-reinforced structures for the purpose of in-situ condition monitoring over the whole service life of a product. Therefore, especially commercially available and tested silicon sensors are well-suited to this end, but new developments can be taken into account as well. The main objective in this work was the development of a smart lightweight semi-finished textile, ready for integration into hybrid structures (i.e. by laminating, injection moulding or vacuum infusion) to consistently predict and monitor faulty behaviour over the service life of the structural part. The integration of the smart textile into a continuous orbital tape winding process illustrates the feasibility of this technology [6]. As shown in figure 1, the smart semi-finished textile consists of a textile substrate tape with integrated stitched or woven electrically conductive fibres, serving as a substrate for a so-called interposer. The interposer is holding the sensors and readout electronics (ASIC), acting as a mechanical protection for downstream integration processes and as an electrical adapter between the microstructures of the sensor system and the mesostructures of the textile. The key technology used for the encapsulation and electrical contacting of the sensor nodes is a two-stage micro injection process, allowing for a cost efficient and application specific mass production. Due to the modular design of the interposer, the same injection moulding tool can be used for a large variety of silicon sensor types.

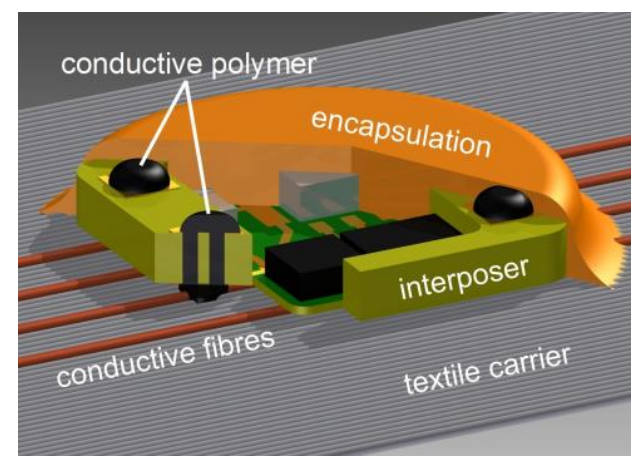

Figure 1: Sketch of the integration concept [4]

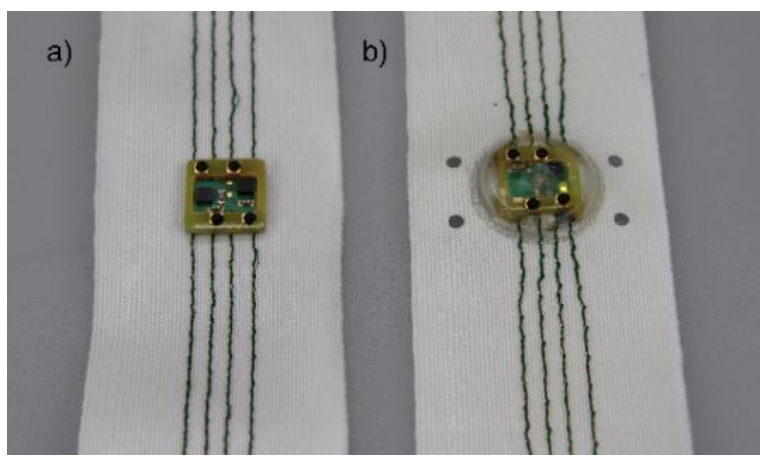

Figure 2: Smart textile without (a) and with (b) encapsulation

\section{Materials and methods}

\subsection{Interposer}

Figure 2 shows a demonstrative part with the developed interposer on a textile tape. In addition to its adapter functionality, the interposer with a modular design allows an easy expansion of the sensor portfolio without the need for redesigning expensive moulding tools. The $10 \times 10 \mathrm{~mm}^{2}$ interposer consists of a multi-layer FR4 PCB with a $0.2 \mathrm{~mm}$ substrate and a $1.2 \mathrm{~mm}$ thick frame. The frame PCB protects the inner electrical components from downstream integration processes. Reflow soldering is used to electrically connect the electrical components on the PCB, whereas in most cases the sensor chips require wire bonding, except for chips using flip chip technology. For a cost-efficient production of the interposer, through-hole plating is possible only on the lower substrate PCB. The electrical connection between upper and lower PCB as well as to the conductive fibres on the textile can be achieved through micro injection moulding of an electrically conductive polymer whose geometry is defined by the injection moulding tool and drill holes. The selection and evaluation of suitable sensor types and especially their read-out electronics made up another important focus. Therefore, three different sensor types (figure 3) were evaluated: a mechanical stress and temperature measurement chip (SMC), an ambient pressure sensor and a three axis acceleration sensor. 


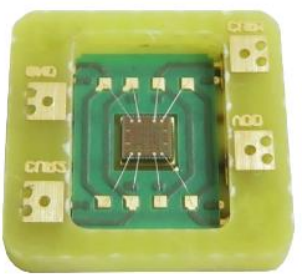

a) SMC

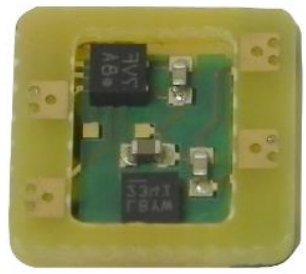

b) Acceleration

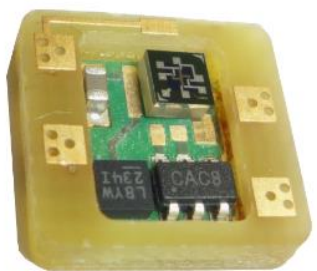

c) Pressure

Figure 3: Selection of sensor types

The stress measurement chip (figure 3a) allows an in-situ determination of stress states before, during and after the assembly steps, i.e. during fabrication and testing of new packages. Additionally, the chip is able to measure the temperature in each cell based on the temperature dependence of the threshold voltage of the NMOS transistors. For this, calibration is required. This system was used for the online measurement of in-plane stress and temperature development during the encapsulation phase. In this investigation we used chips with a size of $1.7 \times 1.8 \mathrm{~mm}^{2}$ and a thickness of $0.2 \mathrm{~mm}$. An array of $6 \times 6$ cells offers 36 measurement points for stress and temperature along the in-plane chip surface with a high read-out rate of $63 \mathrm{~ms}$ per cell.

In addition to the SMC and as a sensor for potential applications of the smart textile we chose the commercially used pressure sensor BMC_SE103 (figure 3c) to measure the pressure as well as outof-plane loads on the chip surface online during the injection moulding process. The sensor has an etched microscopic thin silicon membrane over a sealed cavity with a defined pressure. A change in pressure on the outside of the membrane leads to a deflection in direction of the lower pressure, which can be measured by the help of piezo resistive layers on the membrane. Sensors with a pressure range of 1 bar, 600 bar and 1000 bar were tested. The control and read-out circuit is based on a microcontroller which uses a serial ${ }^{2} \mathrm{C}$ bus.

Furthermore, vibrodiagnostic methods are well-suited for structural health monitoring $[7,8]$. In state-ofthe-art applications piezo or MEMS (Micro Electro Mechanical System) acceleration sensors are placed on the surface of the monitored structure to measure dynamic vibration behaviour. In fibrereinforced composites failure behaviour such as fibre cracking or delamination leads to ultrasonic waves propagating through the solid body, which can be detected by these sensors. In most applications no long-term measurements are possible, whereas continuous long-term measurements can be carried out with the developed smart semi-finished textile. Therefore we selected a three axis acceleration sensor BMA250 developed by Bosch (figure 3b) with an integrated ASIC. It detects acceleration and tilt angles in all three spatial axes.

\subsection{Textile substrate}

The integration of conductor structures was tested on modified versions of established methods of textile technology, such as embroidery, weaving and knitting. The objective was to achieve a flexible processing and integration in fibre composite structures using the textile properties of the tape. In the embroidery process conductive material can be transferred to any textile via a special feed spool. The conductor paths are fixed along the geometry between upper and lower thread (figure 4a) [9]. This technology allows a high flexibility regarding conductor structures, the substrate material, and especially the conductive materials. In addition, this ensures a gentle handling of the filigree conductive fibres. In contrast to embroidery, weaving technology offers the advantage of continuous product manufacturing. In addition, the chosen textile structure with hidden conductor paths protects the conductive fibres against external influences and also improves the component's appearance in near-surface applications. In this substrate for sensor elements in fibre plastic composites, glass fibre yarns and four skeins of copper wires with a defined distance to each other were processed as warp material (figure 4b). The electrically conductive material can be integrated during the knitting process, the result is shown in figure 4c. This technology was very easy to use in terms of cycle times and the flexibility of setting new process parameters. Both the thickness and permeability of the substrate were disadvantageous because of the combination of stitches and the glass fibre yarn as well as the low structural elongation of the conductor material. 


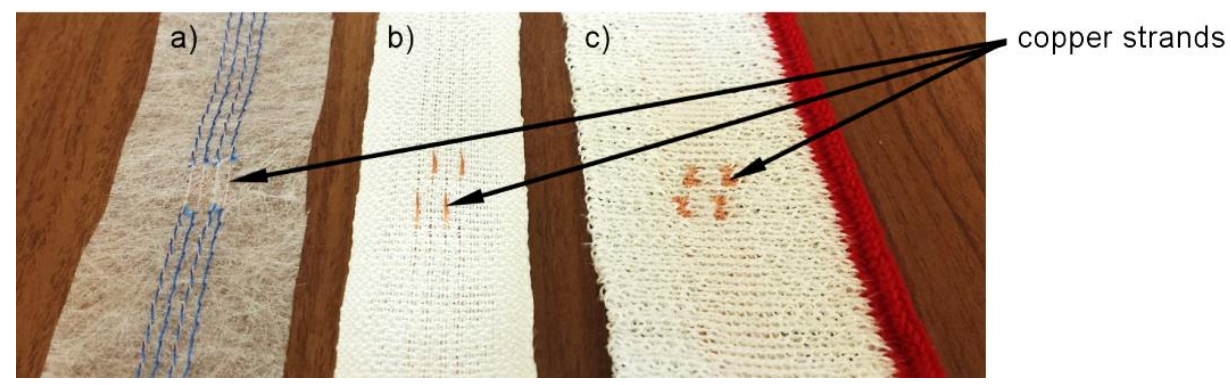

Figure 4: Variously produced textile substrate tapes of glass fibres with integrated conductor paths a) embroidered, b) woven and c) knitted

\subsection{Tool concept for micro injection moulding}

The two-stage two-component micro injection moulding process involves the electrical contacting and encapsulation of the components of sensor networks. In the first step the sensor node is positioned on the textile and electrical contacts are moulded with an electrically conductive polymer using a threeplate injection moulding tool. The cavities for the interposer and the contacting structure are located in a central plate and the gating system is arranged between the central plate and the nozzle side plate. The textile substrate is passed between the ejector side plate and the central plate. Due to this arrangement the sprue is separated from the contacts during the opening of the injection moulding tool. This prevents short-circuiting of the conductive paths through the sprue. In the subsequent injection moulding process the interposer is encapsulated with an insulating polymer and mounted on the textile using another injection moulding tool. The encapsulation polymer can be adapted according to the intended application. The sensor node is mounted on the textile substrate by impregnation of the textile with the encapsulation polymer [10]. The micro injection tools for producing the electrical contacts (a) and the encapsulation (b) of the sensor nodes are shown in figure 5.

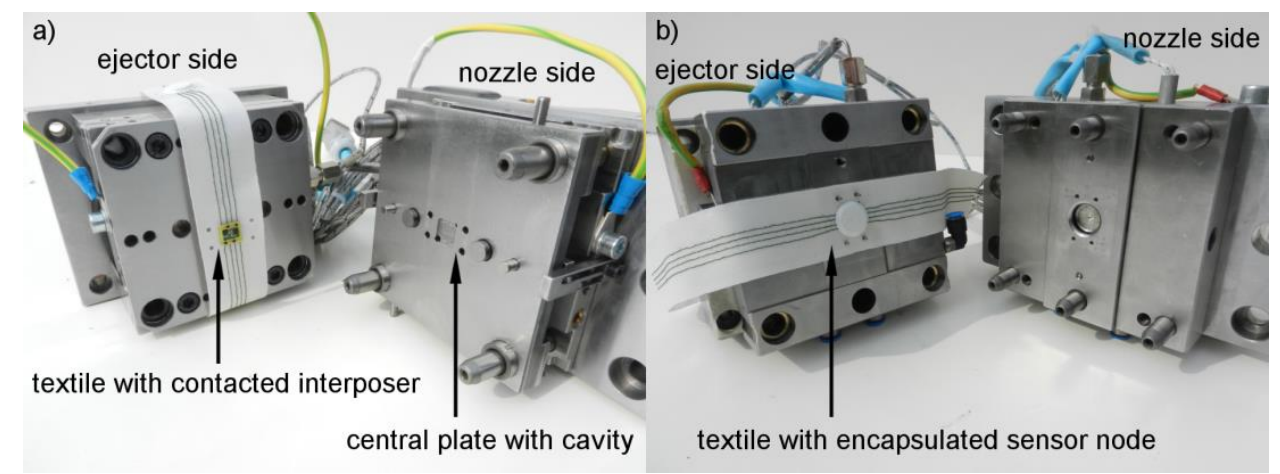

Figure 5: Micro injection moulding tools for a) contacting and b) encapsulation of the sensor nodes

\subsection{Conductive functionalised polymers}

The electrically conductive polymer is based on the ultra-low viscosity polypropylene (PP) homopolymer Moplen HP2774, supplied by LyondellBasell (Rotterdam, The Netherlands). Due to its very high melt flow rate of $1200 \mathrm{~g} / 10 \mathrm{~min}$ (ISO $1133 ; 230^{\circ} \mathrm{C} / 2.16 \mathrm{~kg}$ ) it allows high levels of conductive fillers and a good flowability of the compound. With the aim of an optimised electrical conductivity two different conductive fillers were used. Multi-walled carbon nano tubes (CNT) were the first conductive filler chosen. For this, we used a PP-based masterbatch with $20 \mathrm{wt} \%$ of CNT (Plasticyl PP2001, Nanocyl S.A., Sambreville, Belgium). High conductive carbon black particles (CB) (Ketjenblack EC-600JD, Akzo Nobel GmbH, Düren, Germany) were the second filler. The combination of both fillers shows a lower electrical resistivity than the individual fillers, even at the same filling level. In the compounds with both fillers the volume resistance is dominated by the CNT-loading. With a higher amount of CNT the resistivity decreases exponentially. Analogously, the CB-fraction dominates the contact resistance between the metallic electrodes and the polymer. However, higher amounts of fillers increase the viscosity of the compounds significantly [11]. Based on previous experiments [11] the combination of $8 \mathrm{wt} \% \mathrm{CNT}$ and $8 \mathrm{wt} \% \mathrm{CB}$ is a good compromise between low electrical resistivity, 
low contact resistance and good processability of the compounds by micro injection moulding. Higher filling levels lead to strongly increasing viscosities and thus the compounds cannot be processed.

\subsection{Investigation of the integration processes}

In this study the industrial fabrication process of injection moulding was chosen to prove the integration in harsh process conditions. Therefore, interposers with SMC sensors are used to evaluate the influence of encapsulation processes on the electronic components. Figure 6 depicts the stages from the assembled sensor chip on an organic substrate up to the end of the integration process where the sensor node is completely encapsulated. During this injection moulding process the change of the in-plane stresses and the temperature of the chip were measured in-situ [12].

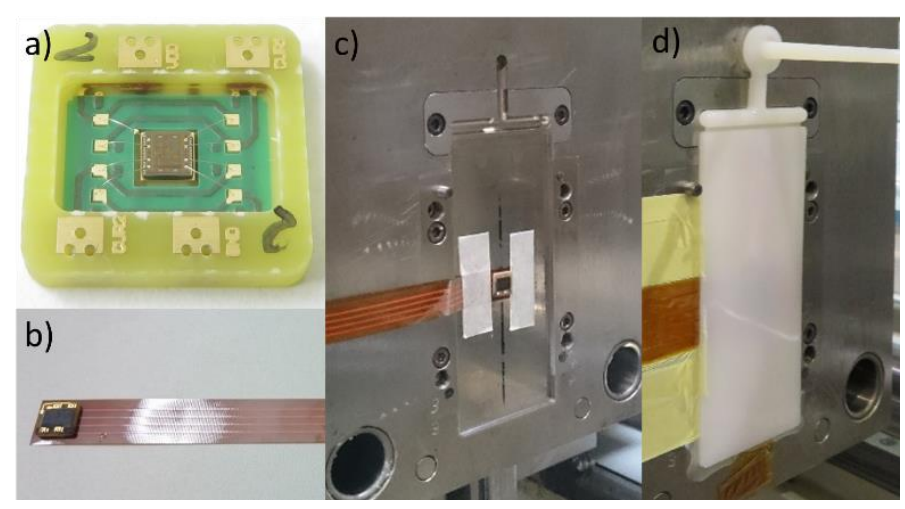

Figure 6: In-situ injection moulding process monitoring with SMC, stages of integration process: a) bare SMC mounted on an interposer, b) encapsulated interposer on a flex substrate, c) sensor node positioned in the cavity before injection moulding, d) integrated sensor in PA6

The first step of the investigation was a feasibility study. Therefore samples with and without a primary encapsulation (glob top) material were used for the integration process. The in-situ temperature and stress of the entire process were successfully measured. The results are shown in figure 7.

a)

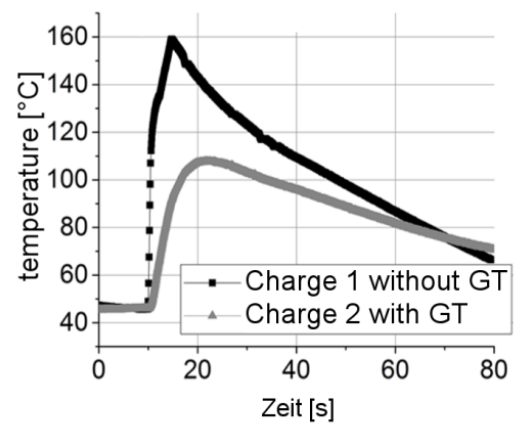

b)

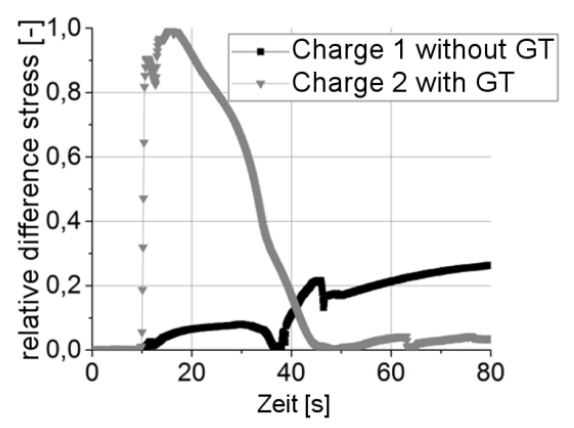

Figure 7: Results of the feasibility study of samples with and without primary encapsulation (glob top) of SMC during injection moulding

Figure $7 \mathrm{a}$ shows the temperature evolution. Ten seconds after the measurement started, the heated tool was closed and the injection began. The temperature increased immediately and reached up to $170{ }^{\circ} \mathrm{C}$ and $110^{\circ} \mathrm{C}$, respectively. The difference is caused by the glob top (GT). The heat flow to the Si chip is reduced when the glob top covers the sensor. Thus, a lower temperature is measured. After $100 \mathrm{~s}$, the injection moulding process was finished and the tool opened. At this time, the final temperature of $60-70{ }^{\circ} \mathrm{C}$ was reached. Correspondingly, figure $7 \mathrm{~b}$ presents the stress induced during the injection moulding process at a typical cell from each of the eight samples. Here, the different inplane stresses are shown. They allow the assessment of the residual stresses inside the package and 
can also be used for the verification of the simulation results. The stress evolution is different for two sample types, i.e. with and without a glob top. The samples with glob top showed a high stress peak of $-160 \mathrm{MPa}$ during the injection starting at $10 \mathrm{~s}$ and the pressure phase finishing at $35 \mathrm{~s}$, which declined rapidly. This is a reaction to the pulse generated by the injected material, which is transferred to the chip surface covered by the glob top. In the following cooling phase, the stresses decrease and reach the state that occurred before the injection moulding process. Statically speaking, the glob top not only lowers but also homogenizes the in-plane stresses induced by the moulding process. By contrast, the samples without a glob top showed a stress impact of less than $10 \mathrm{MPa}$ during injection but one of $40 \mathrm{MPa}$ during the subsequent cooling phase. The molten material did not lead to significant stress while clear differences in static stresses developed along and perpendicular to the direction of injection during solidification. Hence, the glob top material had a significant influence on stress und temperature monitoring. A comprehensive Finite Element Analysis (FEA, ANSYS 17) was performed in addition to experimental tests. The computed lateral mechanical stress distribution was compared to measurement results which were induced by the injection moulding process (figure 8 ). The experiment results, shown in figure $8 \mathrm{a}$, demonstrate the average stress across four samples which range from $8 \mathrm{MPa}$ to $-48 \mathrm{MPa}$. The simulation result matches the experimental findings very closely (see figure $8 b)$. The stress is in the same range (-12 MPa to $-39 \mathrm{MPa})$ and the stress distributions show the same pattern. Additionally, the influence of process parameters has been investigated after the method had been proven by the feasibility study. The cavity temperature was changed from $50{ }^{\circ} \mathrm{C}$ to $100{ }^{\circ} \mathrm{C}$ and the glass fibre filler content of the PA6 melt was varied from $0 \%$ to $30 \%$. The results of this investigation are shown in figure 9.

a)

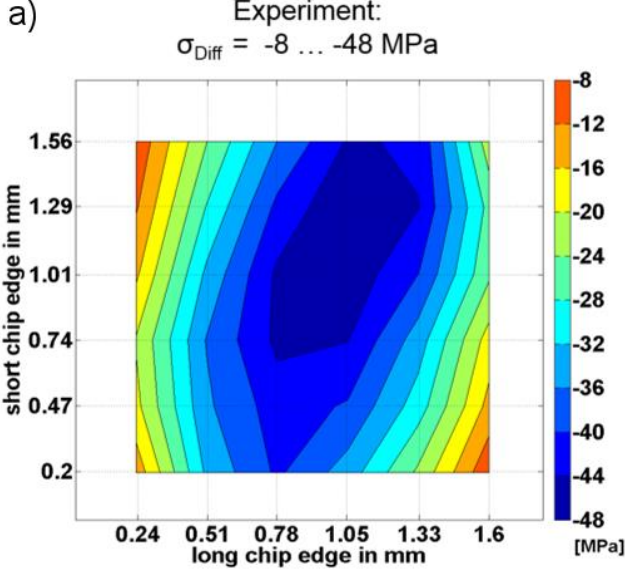

b) Simulation:

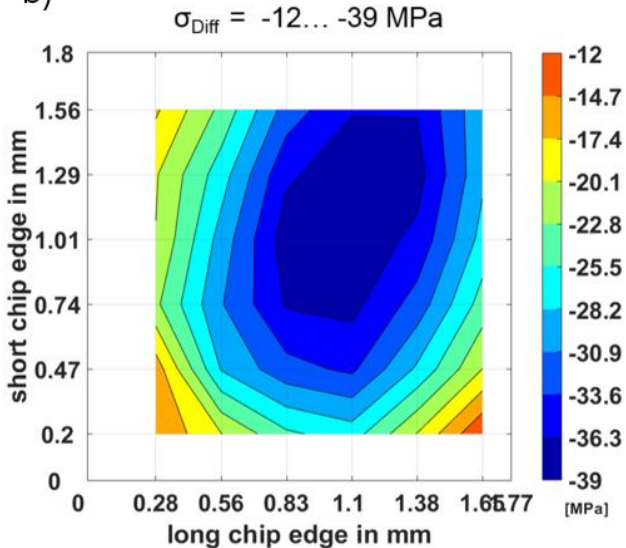

Figure 8: Difference of in-plane stresses induced by injection moulding - experiment (a) and simulation (b)

a)

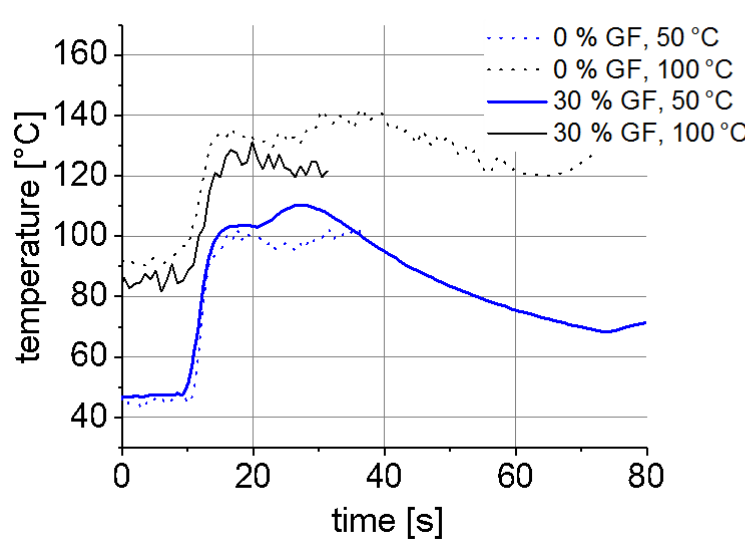

b)

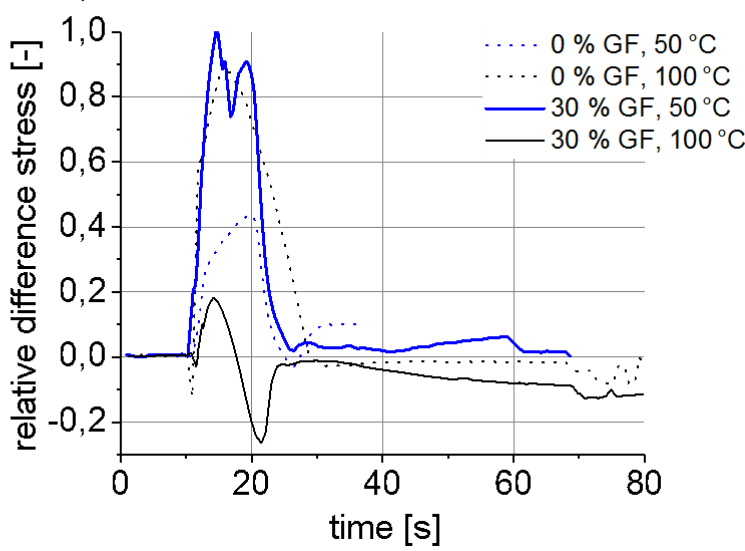

Figure 9: a) Average temperature and b) mechanical stress during injection moulding process with different process parameters of cavity temperature and glass fibre content of PA6 melt 
Figure 9a presents the temperature evolution. The starting temperature equals the cavity temperature. Maximum temperatures of $140^{\circ} \mathrm{C}$ and $110^{\circ} \mathrm{C}$ are measured at the sensor surface during the filling of the $240{ }^{\circ} \mathrm{C}$ melt. Hence there is no critical thermal load on the electronical sensor node. Figure $9 \mathrm{~b}$ shows the mechanical load measured by the SMC. All samples register an input during the filling phase and pressure phase. Afterwards, the loads decrease. An influence of the cavity temperature can be determined. A colder cavity causes a lower stress input on the stress sensor. However, there is no significant influence of the glass fibre filler content on the mechanical stress.

To evaluate the quality of the integration process we performed a cross section and computed tomography (CT) analysis (figure 10).

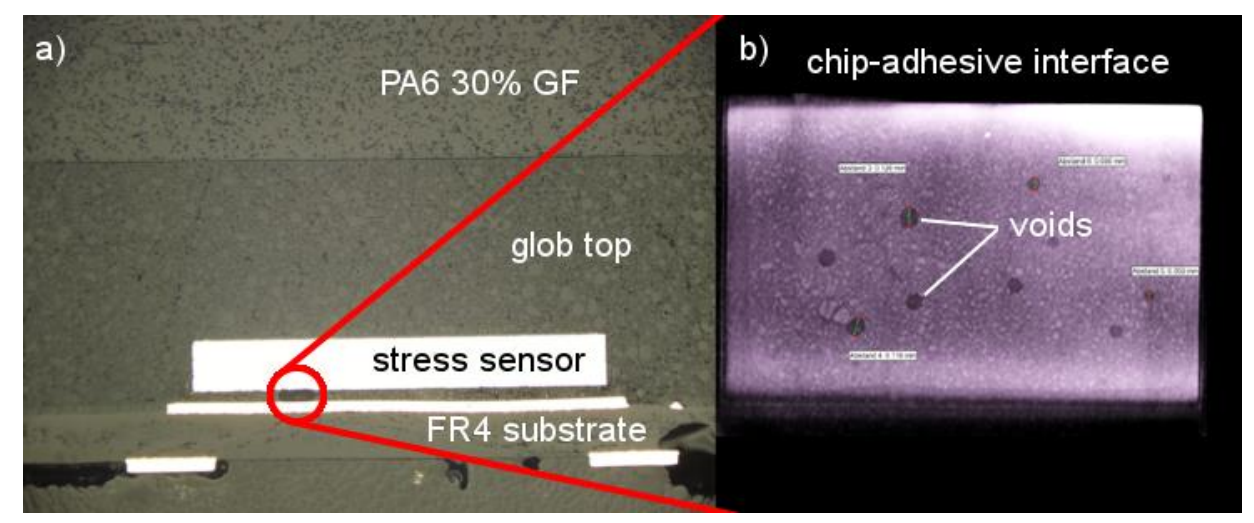

Figure 10: Characterisation of the integrated sensor node by means of a) cross section and b) computed tomography analysis

In the cross section of figure 10a the interfaces between the injection moulding encapsulation and the glob top encapsulation do not show any voids or cracks. The interface between the silicon stress sensor and the glob top material also exhibits good adhesion. Concerning the quality of the chipadhesive interface, the cross section and CT scan (figure 10b) show voids.

\section{Summary and outlook}

In this work, we presented a novel integration concept of silicon sensors into large-scale fibrereinforced semi-finished products with the feasibility of in-situ process and condition monitoring. Different silicon sensor types for the detection of temperature, mechanical stress, acceleration and pressure as well as different textile substrates were evaluated for their use in harsh integration processes. A two-stage two-component micro injection moulding process was chosen for the electrical contacting and encapsulation of the sensor nodes and the influence of filler particles (CNT, CB) on the electrically conductive polymer was studied. As a feasibility study we performed injection moulding experiments using different tool temperatures and filling contents of glass fibre on a SMC sensor node. The results of the in-situ measurements and CT analysis of the specimens match those of our finite element simulations very closely. As a result the smart textile proved to be well-suitable for the monitoring of mechanical stress induced by an injection moulding process. Future works will address studies on the electrical behaviour of the conductive polymer, which are still in progress and could not be presented here in detail. Also, a hybrid textile substrate will be tested for further integration experiments due to its better impregnation properties.

\section{Acknowledgement}

We gratefully acknowledge the cooperation with our project partners and the financial support of the DFG (Deutsche Forschungsgemeinschaft) within the Collaborative Research Center/Transregio 39 "PT-PIESA" and the Federal Cluster of Excellence EXC 1075 "MERGE". 


\section{References}

[1] Oromiehie, E.; Rajan, G.; Compston, P.; Prusty, G. B.: Optical fiber Bragg grating sensors for process monitoring in advanced composites. Sensors and Actuators A: Physical, Volume 103, Issue 3, 15 February 2003, pp. 330-340, DOI: 10.1109/SAS.2016.7479849

[2] Leng, J. S..; Asundi, A.: Structural health monitoring of smart composite materials by using EFPI and FBG sensors. IEEE SENSORS 2014, (2014). DOI: 10.1109/ICSENS.2014.6985315

[3] Schulze, R.; Streit, P.; Fischer, T.; Tsapkolenko, A.; Heinrich, M.; Sborikas, M.; Kroll, L.; Gessner, T.; Wegener, M.: Fiber-reinforced Composite Structures with Embedded Piezoelectric Sensors. IEEE SENSORS 2014, (2014). DOI: https://doi.org/10.1016/S0924-4247(02)00429-6

[4] Schulze, R.; Heinrich, M.; Nossol, P.; Forke, R.; Sborikas, M.; Tsapkolenko, A.; Billep, D.; Wegener, M.; Kroll, L.; Gessner, T.: Piezoelectric P(VDF-TrFE) transducers assembled with micro injection molded polymers. Sensors and Actuators, A: Physical 208 (2014), pp. 159-165.

[5] Sorger, A.; Auerswald, C.; Shaporin, A.; Freitag, M.; Dienel, M.; Mehner, J.: Design, Modeling, Fabrication and Characterization of a MEMS Acceleration Sensor for Acoustic Emission Testing. In: Proceedings of the 17th International Conference on Solid-State Sensors, Acturators and Microsystems, TRANSDUCERS 2013, 2013. DOI: 10.1109/Transducers.2013.6626869

[6] Decker, R.; Arnold, B.; Wallasch, R.; Bauer, A.; Tirschmann, R.; Mehner, J.; Nendel, W.; Kroll, L.: In-line integration of sensors in thermoplastic composite structures using novel continuous orbital winding technology. In: Proceedings of the 21st Symposium on Composites, 2017, pp. 490-497. ISBN: 978-3-0357-1198-1

[7] Nossol, P.; Gliniorz, R.; Kroll, L.; Heinrich, M.: Applications of modal analysis for examining structural state of synthetic-fibre nonwoven composites. Composites Theory and Practice 15 (2015) 3, pp. 130-136.

[8] Auerswald, C.: Mikromechanischer Körperschall-Sensor zur Strukturüberwachung, Dissertation, Technische Universität Chemnitz, 2016

[9] Kroll, L.; Elsner, H.; Heinrich, M.: Sticktechnologische Herstellung von Sensorstrukturen in textilen Trägermaterialien. melliand Textilberichte European Textile Journal (2008) 1-2, pp. 26-27.

[10] Niedziela, D.; Tröltzsch, J.; Latz, A.; Kroll, L.: On the numerical simulation of injection molding processes with integrated textile fiber reinforcements. Journal of Thermoplastic Composite Materials 26 (2013) 1, pp. 74-90. DOI: 10.1177/0892705711419695

[11] Heinrich, M.; Decker, R.; Schaufuss, J.; Troeltzsch, J.; Mehner, J.; Kroll, L.: Electrical contact properties of micro-injection molded Polypropylene/CNT/CB-composites on metallic electrodes. Advanced Materials Research 1103 (2015), pp. 77-83, DOI: 10.4028/www.scientific.net/AMR.1103.77

[12] Rost, F.: Mechanical in-situ characterization of micro systems during encapsulation and integration processes in structural components. In: Proceedings of the 6th Electronics Systemintegration Technology Conference and Exhibition, ESTC2016, 2016 